\title{
SHEAR-WAVE VELOCITY COMPILATION FOR NORTHRIDGE STRONG-MOTION RECORDING SITES
}

\author{
Roger D. Borcherdt and Thomas E. Fumal
}

U.S. Geological Survey Report

Open-File Report Number 2002-107

March 7, 2002

This report is preliminary and has not been reviewed for conformity with U.S. Geological Survey editorial standards or with the North American Stratigraphic Code. Any use of trade, product, or firm names is for descriptive purposes only and does not imply endorsement by the U. S. Government. 


\title{
Shear-Wave Velocity Compilation for Northridge Strong-Motion Recording Sites
}

\author{
Roger D. Borcherdt and Thomas E. Fumal
}

\begin{abstract}
Borehole and other geotechnical information collected at the strong-motion recording sites of the Northridge earthquake of January 17,1994 provide an important new basis for the characterization of local site conditions. These geotechnical data, when combined with analysis of strong-motion recordings, provide an empirical basis to evaluate site coefficients used in current versions of US building codes. Shear-wave-velocity estimates to a depth of 30 meters are derived for 176 strong-motion recording sites. The estimates are based on borehole shear-velocity logs, physical property logs, correlations with physical properties and digital geologic maps. Surface-wave velocity measurements and standard penetration data are compiled as additional constraints. These data as compiled from a variety of databases are presented via GIS maps and corresponding tables to facilitate use by other investigators.
\end{abstract}

\section{INTRODUCTION}

Ground shaking generated by the Northridge earthquake of January 17,1994 was recorded by strong-motion instruments at more than 200 sites located out to distances of $250 \mathrm{~km}$. Horizontal ground acceleration at the surface exceeded $0.9 \mathrm{~g}$ at seven of these sites within 22 $\mathrm{km}$ of the epicenter and $0.4 \mathrm{~g}$ at 31 sites within $40 \mathrm{~km}$ of the epicenter. As the largest nearsource, strong-motion data set yet collected in the US, these recordings provide an important basis for empirical estimates of site-specific amplification factors $F_{a}$ and $F_{v}$ as specified in current US building codes at input ground motion levels greater than $0.1 \mathrm{~g}$ (Borcherdt, 2002a and 2002b).

Detailed geotechnical data collected over a period of several years both before and since the Northridge earthquake provides the necessary basis to characterize the local site conditions quantitatively at the recording sites. These data are needed to quantify the site conditions using shear velocity to $30 \mathrm{~m}$ depth and in turn classify the sites according to siteclass definitions used in present versions of building codes. This report provides a compilation of shear-wave velocity estimates to a depth of $30 \mathrm{~m}$ for 176 strong-motion sites. Estimates are derived from borehole seismic and physical property logs, correlations with physical properties, digital geologic maps as displayed using a GIS, and additional information as guidelines.

Site characterizations herein are based on information assembled from databases kindly provided by a number of investigators (Boore, pers. commun. 1995; Silva, pers. commun. 1995; Vucetic, pers. commun. 1996, Wills and Silva, pers. commun. 1998; Nigbor, and Bardet; ROSRINE, 1999; Gibbs et al., 1999; King, pers. commun. 1995). These data compiled in GIS as reviewed with site visits by Fumal are used to provide a relatively complete and up-to-date list of shear-velocity estimates to $30 \mathrm{~m}$ for the recording sites. 


\section{STRONG-MOTION SITE CHARACTERIZATION IN TERMS OF $V_{S_{30}}$}

Borehole logging efforts to compile shear-wave velocity logs and corresponding physical property $\operatorname{logs}$ to depths of at least of $30 \mathrm{~m}$ provide the desired database for characterizing the strong-motion sites. The major borehole data sets as collected both before and after the Northridge earthquake by Gibbs et al. (1980; 1996), Fumal et al., (1981; 1982a; 1982b; 1984), and Nigbor et al., (1998) are tabulated in databases of Boore et al. (1995, pers. commun.), Bardet, et al. (1998, http://rccg03.usc.edu/rosrine/), Silva, (1998, pers. commun.), Wills and Silva (1998), and Vucetic, et al. (1996, pers. commun.). Many of the boreholes are near the strong-motion instrument sites and hence, provide a measurement that can be used as an accurate estimate of $v_{s_{30}}$. In other cases the boreholes are not near the recording site and some type of extrapolation is needed.

Towards developing estimates of the shear-wave velocity at sites for which no borehole measurements were nearby, geotechnical information was compiled from digital files of mapped surface geology and site-specific digital databases. Surface geology as compiled digitally from Tinsley and Fumal (1985), Yerkes (pers. commun, 1994), and Jennings, et al., (1977) were used to compile data in a geographic information system (GIS). Digital databases compiled by Boore, (pers. commun. 1996), Silva, (pers. commun, 1998), and Vucetic and Doroudian, (1995) with modifications by King (pers. commun. 1996) were compiled in GIS to provide additional site-specific information. The various geologic maps were displayed using GIS at large scales with superimposed locations labeled with estimates of $v_{s}$ to $30 \mathrm{~m}$ as derived from boreholes and surface wave measurements. Working versions of these maps were used to infer the spatial relationships of the various data sets and derive

estimates of $v_{s_{30}}$ for the strong-motion recording sites. $v_{s}$ measurements as inferred from borehole and surface wave measurements are shown in Figure 1 superimposed on the digital geologic database of Tinsley-Fumal (1985) and in Figure 2 as superimposed on the digital geologic database of Jennings, et al., (1977). (Similar maps are available on request using the site condition map of Wills et al. (2001) as a base.)

Shear-wave velocity estimates for the strong-motion recording sites are tabulated in Table 1. Five types of estimates are indicated. "Type 1" estimates are those derived from borehole measurements within $300 \mathrm{~m}$ of the location of the strong-motion recording by the USGS (Gibbs, et al., 1980, 1996, 1999, 2000) or Agbabian and associates (Nigbor, 1998) as part of the ROSRINE program. These data are available in databases developed by Boore (1995, pers. commun.), Bardet et al. (1998), Silva (pers. commun. 1998), and Vucetic and Doroudian (1995). "Type 1b" estimates are derived from borehole measurements within 300 $\mathrm{m}$ of the site by CDMG, NUREG, or Crandall and Associates as compiled in the databases of Silva and Vucetic. "Type 2" estimates were derived from borehole measurements from one of the sources identified as "Type 1 or $1 \mathrm{~b}$ ", but up to distances of $1500 \mathrm{~m}$ from the site. "Type 3" identifies those estimates derived from averages computed from measurements in other boreholes in materials with similar physical properties (Fumal, 1978; Fumal and Tinsley, 1985). Sites used to derive the Type 3 estimates are tabulated. "Type 4" identifies those estimates derived from average velocity estimates derived herein for the corresponding geologic unit. Standard deviations for the samples used to Type 4 estimates are tabulated. "Type 5" refers to measurements inferred using surface wave techniques as summarized in the databases of Silva and Vucetic. Type 1 or 2 estimates are available for nearly all of the sites with base accelerations exceeding $0.3 \mathrm{~g}$ near or within the projected rupture surface. 
Surface geology classifications are provided for each of the sites in Table 1. Sites were classified using physical property logs if available for Type 1 and $1 \mathrm{~b}$ sites, onsite inspections, for sites visited, and detailed geologic digital maps if other information was not available. Site class designation was assigned to each site based on the estimate of $v_{s_{30}}$.

A histogram showing the distribution of shear-wave velocity estimates for the strongmotion recording sites is shown in Figure 3a. The corresponding histogram for only sites with borehole measurements (type 1 and $1 \mathrm{~b}$ sites) is shown in Figure 3b. The histogram for all sites suggests a tri-modal distribution with local maximum at $337 \mathrm{~m} / \mathrm{s}, 437 \mathrm{~m} / \mathrm{s}$ and 862 $\mathrm{m} / \mathrm{s}$. This tri-modal distribution is consistent with the site class definitions used in present building code provisions. The histograms (Figures $3 \mathrm{a}$ and $3 \mathrm{~b}$ ) indicate gradual transitions in material characteristics at site class boundaries. They show that materials in site classes B, C, and $\mathrm{D}$ underlie the strong-motion recording sites with shear-wave velocities ranging between 200 and $1300 \mathrm{~m} / \mathrm{s}$.

\section{COMPARISON OF BOREHOLE $V_{S_{30}}$ AND SURFACE-WAVE ESTIMATES}

Shear-wave velocity measurements inferred using noninvasive surface-wave techniques are less expensive and generally easier to obtain than borehole measurements. However, uncertainties introduced by the need to infer material characteristics at depth using a variety of models and model assumptions have tended to discourage their use. Comparisons of shear velocity estimates derived from surface wave information and those derived from borehole measurements are shown in Figures $4 \mathrm{a}$ and $4 \mathrm{~b}$. Comparisons are provided for sites with type 1 borehole data as well as those with types 1, 2, 3, and 4 data. Best fitting least squares lines and correlation coefficients are shown for each comparison together with a theoretical line with unity slope and exact correlation.

The comparisons (Figures $4 \mathrm{a}$ and $4 \mathrm{~b}$ ) indicate significant variation in some of the surface wave inferences. The correlation between surface wave measurements and those derived from borehole data is better for Type 1 borehole estimates as opposed to all of the Type 1, 2, 3 , and 4 data. However, the Type 1 sample with surface wave data is also significantly smaller. With the exception of site RPV for which the surface wave measurement appears quite anomalous and has been eliminated from the plot, the comparisons indicate that the surface wave measurements show a well-defined correlation with those inferred from borehole information. This comparison suggests that the relationships such as those indicated in Figures $4 \mathrm{a}$ and $4 \mathrm{~b}$ together with surface wave measurements would indeed be useful for characterizing site conditions at sites for which borehole measurements are not feasible.

\section{ACKNOWLEDGMENTS}

Site condition data compiled by a number of investigators and assembled in various databases were critical for this study. Databases compiled by Dave Boore, Walt Silva, and M. Vucetic with modifications by Stephanie King and Chris Wills were kindly provided via personal communication. "This manuscript incorporates information from the Preliminary Statewide Site Condition Map of California (PSSCM), which is protected by the United States Copyright Law. The PSSCM can only be reproduced with the written consent of the State of California. For information contact the California Department of Conservation. A careful review of the manuscript by J. Tinsley was helpful. 


\section{REFERENCES}

Bardet, J. P. Nielsen, E. and Villacorta, R. 1998, ROSRINE Data Dissemination, http://geoinfo.usc.edu/rosrine/Publications/jpbardet/, 2/28/2002.

Borcherdt, R.D., 1992, Simplified site classes and empirical amplification factors for site-dependent code provisions, in, Proceedings NCEER, SEAOC, BSSC workshop on site response during earthquakes and seismic code provisions, G. Martin (editor), University of Southern California, Los Angeles, California, November 18 - 20, 1992.

Borcherdt, R.D. 1994. Estimates of site-dependent response spectra for design (Methodology and Justification), Earthquake Spectra, 10, p. 617-653.

Borcherdt, R.D., 1996, Preliminary amplification estimates inferred from strong ground-motion recordings of the Northridge earthquake of January 17, 1994, International Workshop on Site Response Subjected to Strong Earthquake Motions, Procs., Port and Harbor Research Institute, January 16 - 17, 1996, Yokosuka, Japan, v I, p24-33 and v II, pp. 21-46.

Borcherdt, R. D., 2002, Empirical evidence for acceleration-dependent amplification factors, Bull. Seismol. Soc. Am., accepted.

Borcherdt, R. D., 2002, Empirical evidence for site coefficients in building-code provisions, Earthquake Spectra, accepted.

Fumal, T.E. 1978. Correlations between seismic wave velocities and physical properties of geologic materials in the San Francisco Bay region, California, U.S. Geological Survey Open-File Report 78-1067.

Fumal, T.E., Gibbs, J.F., and Roth, E.F., 1981, In-situ measurements of seismic velocity at 19 locations in the Los Angeles, California region: U.S. Geological Survey Open-File Report 81-399, p. $1-121$.

Fumal, T.E., Gibbs, J.F., and Roth, E.F., 1982, In-situ measurements of seismic velocity at 10 strong motion accelerograph stations in central California: U.S. Geological Survey Open-File Report 82407, p. $1-76$.

Fumal, T.E., Gibbs, J.F., and Roth, E.F., 1982, In-situ measurements of seismic velocity at 22 locations in the Los Angeles, California region: U.S. Geological Survey Open-File Report 82-833, p. 1-138.

Fumal, T.E., Gibbs, J.F., and Roth, E.F., 1984, In-situ measurements of seismic velocity at 16 locations in the Los Angeles, California region: U.S. Geological Survey Open-File Report 84681, p. 1-109.

Fumal, T.E. and Tinsley, J.C. 1985. Mapping Shear-wave velocities of near-surface geologic materials. U.S. Geological Survey Professional Paper 1360, p. 127-150.

Gibbs, J.F., Fumal, T.E., and Roth, E.F., 1980, In-situ measurements of seismic velocity at 27 locations in the Los Angeles, California region: U.S. Geological Survey Open-File Report 80-378, p. 1-167.

Gibbs, J. F., Tinsley, J. C., and Joyner, W. B., 1996, Seismic velocities and geological conditions at twelve sites subjected to strong ground motion in the 1994 Northridge California, earthquake: U.S. Geological Survey Open-File Report 96-740, 103 pp.

Gibbs, J. F., Tinsley, J. C., Boore, D. M., and Joyner, W. B., 1999, Borehole velocity measurements and geological conditions at thirteen sites in the Los Angeles, California region,: U.S. Geological Survey Open-File Report 00-470, 118 pp.

Gibbs, J. F., Tinsley, J. C., Boore, D. M., and Joyner, W. B., 2000, Seismic velocities and geological conditions at twelve sites subjected to strong ground motion in the 1994 Northridge California, earthquake: a revision of OFR 96-740: U.S. Geological Survey Open-File Report 99-446, 142 pp.

Jennings, C. W., Strand, R. G., and Rogers, T. H., 1977, Geologic map of California, California Division of Mines and Geology, Sacramento, CA.

Nigbor, R., 1998, ROSRINE Field Investigations, http://rccg03.usc.edu/rosrine/Publications/bnigbor/. 
Porcella, R.L., Etheridge, E.C., Maley, R.P., and Acosta, A.V., 1994, Accelerograms recorded at USGS national strong-motion network stations during the Ms=6.6 Northridge, California earthquake of January 17, 1994, U. S. Geological Survey Open-File Report 94-141, 100 pp.

Shakal, A., Huang, M., Darragh, R., Cao, T., Sherburne, R., Malhotra, P., Cramer, C., Sydnor, R., Grazier, V., Maldonado, G., Petersen, C., and Wampole, J., 1994, CSMIP Strong-motion records from the Northridge, California earthquake of January 17, 1994, California Strong-Motion Instrumentation Program Report Number OSMS 94-07, 308 pp.

Tinsley, J.C. and Fumal, T.E., 1985, Mapping Quaternary sedimentary deposits for aerial variations in shaking response, in, Evaluating earthquake hazards in the Los Angeles region - an earth scienc3eperspoective; J. I. Ziony (editor), U. S. Geological Survey Professional Paper 1360, 101126.

Trifunac, M.D., Todorovska, M.I., and Ivanovic', S.S., 1994, A note on the distribution of uncorrected peak ground accelerations during the Northridge earthquake of 17 January 1994, University of Southern California.

Vucetic, M. and Doroudian, M., 1995, Task H-5: geotechnical site data base for southern California", Final Task Report on "The Characteristics of Earthquake Ground Motions for Seismic Design", Southern California Earthquake Center - SCEC, University of Southern California, 1, 2, and 3, 1546 pp.

Wills, C.J., Petersen, M., Bryant, W. A., Reichle, M., Saucedo, G.J., Tan, S., Taylor, G. and Treiman J. (2001). A site conditions map for California based on geology and shear wave velocity, scale 1:250,000, California Division of Mines and Geology, Sacramento, CA. 
Table 1. Identification, surface geology, and shear-wave velocity inferred from boreholes and surface-wave measurements for strong-motion stations that recorded the Northridge earthquake.

\begin{tabular}{|c|c|c|c|c|c|c|c|c|c|c|c|}
\hline & & & & Geolos & & & & Shear-w & Velocity & & \\
\hline Station ID & & & $\begin{array}{l}\text { Coordinate } \\
\text { s }\end{array}$ & Description & Symbol & & & $v_{s b h}$ & $V_{s s w}$ & Site & \\
\hline Name & code & Long. & Lat. & & & type ${ }^{*}$ & $\mathrm{~m} / \mathrm{s}$ & SD type4 & $\mathrm{m} / \mathrm{s}$ & Class & Notes \\
\hline Sylmar Conv. Sta East & SCE & -118.481 & 34.312 & Pleistocene alluv (Saugus) & Ts & 1 & 366 & & & C & \\
\hline Jensen Plant, Admin. & JFP & -118.496 & 34.312 & Fill/Hol medium alluvium & Qym & 1 & 373 & & & C & \\
\hline Jensen Plant, Gener. & $\begin{array}{l}\text { JFP } \\
G\end{array}$ & -118.498 & 34.313 & Pleistocene alluv (Saugus) & Ts & 1 & 526 & & & C & \\
\hline Sylmar Converter Sta 7 & SC7 & -118.490 & 34.311 & Holocene fine alluvium & Qyf & 1 & 251 & & & $\mathrm{D}$ & \\
\hline Sylmar Prk Lot & SYL & -118.444 & 34.326 & Holocene coarse alluvium & Qyc & 1 & 441 & & & C & \\
\hline Rinaldi Recv Sta & $\mathrm{RIN}$ & -118.479 & 34.281 & Holocene medium alluvium & Qym & 1 & 333 & & & $\mathrm{D}$ & \\
\hline Newhall & NWH & -118.530 & 34.390 & Alluvium & Qa & 1 & 269 & & & D & \\
\hline Newhall Sun Oil-Potrero 1 & NWS & -118.622 & 34.391 & $17.5 \mathrm{~m}$ alluv/Pliocene siltstone & $\mathrm{Q} a / T p$ & 1 & 282 & & & $\mathrm{D}$ & \\
\hline Pacoima Dam & PCD & -118.396 & 34.334 & Diorite gneiss & gn & 3 & 880 & & & B & Warm Springs site $75^{7}$ \\
\hline Pacoima Kagel & PKC & -118.375 & 34.296 & Tertiary sandstone & & 1 & 509 & & & C & \\
\hline Sepulveda VA Hosp & VSP & -118.475 & 34.249 & Pleistocene fine alluvium & Qof & 1 & 365 & & & C & \\
\hline Arleta & ARL & -118.439 & 34.236 & Holocene coarse alluvium & Qyc & 1 & 302 & & & $\mathrm{D}$ & \\
\hline Sun Valley Ch & SVG & -118.422 & 34.221 & Holocene medium alluvium & Qym & 4 & 318 & 79 & 385 & $\mathrm{D}$ & \\
\hline Canyon Country & $\mathrm{CCY}$ & -118.426 & 34.419 & Holocene medium alluvium & Qym & 4 & 318 & 79 & & $\mathrm{D}$ & \\
\hline Northridge -White Oak Ch & $N R G$ & -118.517 & 34.209 & Holocene medium alluvium & Qym & 1 & 281 & & 269 & $\mathrm{D}$ & \\
\hline North Hollywood C Sch & $\mathrm{NHW}$ & -118.412 & 34.194 & Holocene coarse alluvium & Qyc & 4 & 448 & 47 & 399 & C & \\
\hline Simi Valley- Knolls Elm Sch & SMI & -118.666 & 34.264 & Holocene medium alluvium & Qym & 1 & 561 & & 308 & C & \\
\hline LA UCLA Gnds & UCG & -118.439 & 34.068 & Pleistocene medium alluvium & Qom & $1 b$ & 437 & & & C & \\
\hline $\begin{array}{l}\text { Canoga Park-Epiphany Lut } \\
\text { Ch }\end{array}$ & $\mathrm{CPC}$ & -118.606 & 34.212 & Holocene medium alluvium & Qym & 1 & 283 & & 282 & $\mathrm{D}$ & \\
\hline Tarzana & TAR & -118.534 & 34.160 & Mid-Late Miocene siltstone/shale & $\mathrm{Tm}$ & 1 & 302 & & & $\mathrm{D}$ & \\
\hline Sunland Gleason Sch & SUN & -118.303 & 34.269 & Holocene coarse alluvium & Qyc & 4 & 448 & 47 & 361 & C & \\
\hline Santa Susana ETEC & SSA & -118.713 & 34.231 & Cretaceous sandst(Chatsworth) & $\mathrm{Kc}$ & 1 & 719 & & & C & \\
\hline LA F Sta 99 & LF6 & -118.440 & 34.132 & M-Late Miocene sandst (Monterey) & Tmss & 4 & 492 & 153 & 276 & C & \\
\hline Burbank C Rest & $\mathrm{BCY}$ & -118.302 & 34.204 & Granitic rock & gr & 4 & 828 & 115 & 874 & B & \\
\hline
\end{tabular}




\begin{tabular}{|c|c|c|c|c|c|c|c|c|c|c|c|}
\hline LA F Sta 108 & LF5 & -118.405 & 34.127 & Mid-Miocene sandst(Mid-Topanga) & Tts & 3 & 780 & & 374 & B & Devils Punchbowl ${ }^{9}$ \\
\hline La Crescenta & LCA & -118.254 & 34.238 & Holocene v coarse alluvium & Qyvc & 4 & 448 & 47 & 319 & C & \\
\hline Castaic Old Ridge Route & ORR & -118.642 & 34.564 & Lt-Miocene mudst/sandst(Castaic) & Mc & 2 & 451 & & & C & \\
\hline $\begin{array}{l}\text { LA Wonderland Ave Elem } \\
\text { Sch }\end{array}$ & LWE & -118.380 & 34.115 & Granitic rocks & gr & 1 & 1270 & & 1235 & $\mathrm{~B}$ & \\
\hline Lake Hughes Sta 12A & L12A & -118.560 & 34.571 & Hol coarse al/Eocene sandst & Qyc/Esf & 1 & 600 & & & $\mathrm{C}$ & \\
\hline Topanga F Sta & TOP & -118.599 & 34.084 & Landsld/Low-Mio ss(Low-Topanga) & Ttlsc & 3 & 780 & & & $B$ & Devils Punchbowl $^{9}$ \\
\hline LA St Mary's Sch & MSM & -118.482 & 34.086 & Santa Monica slate & sms & 3 & 650 & & 606 & C & \\
\hline LA Westlake Sch & LWS & -118.435 & 34.090 & Pleistocene medium alluvium & Qom & 4 & 414 & 85 & 368 & C & \\
\hline Big Tujunga Station & BTS & -118.225 & 34.286 & Holocene very coarse alluvium & Qyvc & 4 & 448 & 47 & 423 & C & \\
\hline Vasquez Rock Park & VRP & -118.327 & 34.492 & 3m Aluv/Olig.sandstone(Vasquez) & Tvss & 3 & 780 & & & $\mathrm{~B}$ & Devils Punchbowl ${ }^{9}$ \\
\hline Glendale F Sch & GLF & -118.231 & 34.200 & Holocene coarse alluvium & Qyc & 4 & 448 & 47 & 239 & C & \\
\hline Hollywood Strg Lot FF & HSL & -118.339 & 34.090 & Holocene fine alluvium & Qyf & 2 & 318 & & & $\mathrm{D}$ & \\
\hline Century City North & $\mathrm{CCN}$ & -118.418 & 34.063 & Pleistocene medium alluvium & Qom & $1 b$ & 302 & & & $\mathrm{D}$ & \\
\hline Hollywood Child C & HLC & -118.365 & 34.088 & Holocene fine alluvium & Qyf & 4 & 240 & 56 & 360 & $\mathrm{D}$ & \\
\hline LA F Sta 23 & LF3 & -118.554 & 34.042 & Holocene coarse alluvium & Qyc & 4 & 448 & 47 & 348 & C & \\
\hline $\begin{array}{l}\text { Lake Hughes Sta } 9 \text { Warm } \\
\text { Sp }\end{array}$ & L09 & -118.558 & 34.608 & Sawtooth gneiss & sgn & 1 & 882 & & & $\mathrm{~B}$ & \\
\hline Santa Monica City Hall & SMC & -118.490 & 34.011 & Pleistocene medium alluvium & Qom & 2 & 426 & & & C & Santa Monica site $52^{6}$ \\
\hline Moorpk. L. Hughes Arr & MPK & -118.881 & 34.288 & Pleistocene medium alluvium & Qom & 4 & 414 & 85 & & C & \\
\hline Griffith Park Obs. & GPK & -118.299 & 34.118 & Granodiorite & gr & 1 & 980 & & & $\mathrm{~B}$ & \\
\hline LA Dayton Hgts Sch & LDH & -118.298 & 34.082 & Up-Miocene siltstone(Puente) & Tpsl & 1 & 330 & & 381 & $\mathrm{D}$ & \\
\hline LA F Sta 50 & LF1 & -118.244 & 34.115 & Holocene coarse alluvium & Qyc & 4 & 448 & 47 & 294 & C & \\
\hline LA Saturn St Sch & LSS & -118.355 & 34.047 & Holocene fine alluvium & Qyf & 1 & 296 & & 264 & $\mathrm{D}$ & \\
\hline LA McBride Sch & MBS & -118.431 & 34.001 & Holocene fine alluvium & Qyf & 4 & 240 & 56 & 330 & $\mathrm{D}$ & \\
\hline Glendora Ch 120 & GMC & -117.882 & 34.137 & Holocene coarse alluvium & Qyc & 4 & 448 & 47 & 348 & C & \\
\hline Baldwin Hills & $\mathrm{BHL}$ & -118.361 & 34.009 & Shale, sandstone & Qom & 1 & 293 & & & $\mathrm{D}$ & \\
\hline LA Temple and Hope & LAT & -118.246 & 34.059 & Up-Miocene siltstone(Puente) & Tpsl & 2 & 368 & & & C & avg 3 sites (LC) \\
\hline Lake Hughes Sta 4 & L04 & -118.478 & 34.650 & Wthrd granite & gr & 4 & 828 & 115 & & $\mathrm{~B}$ & \\
\hline Lake Hughes Sta 4B & L4B & -118.477 & 34.650 & Hol med alluv/wthrd granite & Qym/gr & 1 & 351 & & & $\mathrm{D}$ & \\
\hline LA St Thomas Sch & LST & -118.298 & 34.045 & Pleistocene medium alluvium & Qom & 4 & 414 & 85 & 244 & C & \\
\hline Pico \& Sentous & $\mathrm{PIC}$ & -118.271 & 34.043 & Holocene fine alluvium & Qyf & 2 & 326 & & & $\mathrm{D}$ & \\
\hline LA Divine Saviour Sch & LDS & -118.222 & 34.088 & Holocene coarse alluvium & Qyc & 4 & 448 & 47 & 407 & C & \\
\hline LA F Sta 12 & LF2 & -118.189 & 34.113 & Pleistocene medium alluvium & Qom & 4 & 414 & 85 & 374 & C & \\
\hline Playa Del Rey & PDR & -118.432 & 33.960 & Pleistocene medium alluvium & Qom & 4 & 414 & 85 & 486 & C & \\
\hline Univ Hosp & UHS & -118.198 & 34.062 & Up-Miocene siltstone(Puente) & Tpsl & $1 b$ & 312 & & & $\mathrm{D}$ & \\
\hline
\end{tabular}




\begin{tabular}{|c|c|c|c|c|c|c|c|c|c|c|c|}
\hline unknown & UNK & -118.290 & 34.020 & Holocene medium alluvium & Qym & 4 & 318 & 79 & 305 & $\mathrm{D}$ & \\
\hline San Marino & SNM & -118.130 & 34.115 & Pleistocene coarse alluvium & Qoc & 3 & 650 & & & $\mathrm{C}$ & Katella site $11^{3}$ \\
\hline Pt. Dume Sch Malibu & PDS & -118.800 & 34.013 & M-Late Miocene shale(Monterey) & $\mathrm{Tm}$ & 3 & 373 & & & $\mathrm{C}$ & avg sites $39^{4}, 40^{4}, 45^{4}, \mathrm{TAR}^{10}$ \\
\hline Alhambra & ALF & -118.150 & 34.070 & Pleistocene medium alluvium & Qom & 3 & 419 & & 550 & C & Alhambra site $47^{6}$ \\
\hline Mt Wilson & MTW & -118.057 & 34.224 & Quartz diorite & qd & 4 & 828 & 115 & & $\mathrm{~B}$ & \\
\hline Lake Hughes Sta 1 & L01 & -118.430 & 34.674 & Hol med alluv/wthrd granite & Qym/gr & 1 & 424 & & & C & \\
\hline Camarillo LH Array & $\mathrm{CMO}$ & -119.079 & 34.208 & Holocene fine alluvium & Qyf & 4 & 240 & 56 & & $\mathrm{D}$ & \\
\hline Elizabeth Lake & ELK & -118.387 & 34.662 & Holocene fine alluvium & Qyf & 4 & 240 & 56 & & $\mathrm{D}$ & \\
\hline Leona Valley Sta 2 & LV2 & -118.243 & 34.595 & Pleistocene very coarse aluvium & Qovc & 4 & 486 & 37 & & $\mathrm{C}$ & \\
\hline Leona Valley 3 & LV3 & -118.243 & 34.596 & Plio-Pleistocene v. coarse aluvium & QTcs & 3 & 628 & & & $\mathrm{C}$ & LA Dam ${ }^{8}$ \\
\hline Obregon Park & $\mathrm{OBG}$ & -118.178 & 34.037 & Pleistocene medium alluvium & Qom & 1 & 348 & & & $\mathrm{D}$ & \\
\hline Leona Valley Sta 4 & LV4 & -118.242 & 34.598 & Pleistocene very coarse alluvium & Qovc & 4 & 486 & 37 & & $\mathrm{C}$ & \\
\hline Leona Valley Sta 5 & LV5 & -118.241 & 34.600 & Holocene coarse alluvium & Qyc & 4 & 448 & 47 & & C & \\
\hline Anaverde Valley & AVY & -118.199 & 34.580 & Holocene coarse alluvium & Qyc & 4 & 448 & 47 & & $\mathrm{C}$ & \\
\hline Leona Valley Sta 6 & LV6 & -118.244 & 34.604 & Pleistocene coarse alluvium & Qoc & 4 & 466 & 111 & & $\mathrm{C}$ & \\
\hline Pasadena SMV Ave & SMV & -118.078 & 34.169 & Holocene coarse alluvium & Qoc & 4 & 466 & 111 & 291 & C & \\
\hline LA W V Sch & LVS & -118.279 & 34.005 & Holocene medium alluvium & Qym & 4 & 318 & 79 & 296 & $\mathrm{D}$ & \\
\hline Vernon City Sch & VCS & -118.230 & 34.004 & Holocene medium alluvium & Qym & 4 & 318 & 79 & 319 & $\mathrm{D}$ & \\
\hline Downey South Middle Sch & DWY & -118.137 & 33.920 & Holocene medium alluvium & Qym & 1 & 250 & & 254 & $\mathrm{D}$ & \\
\hline San Gabriel Lin Sch & SGS & -118.093 & 34.092 & Pleistocene medium alluvium & Qom & 4 & 414 & 85 & 693 & $\mathrm{C}$ & \\
\hline LA 116th Str Sch & LAS & -118.260 & 33.929 & Pleistocene medium alluvium & Qom & 4 & 414 & 85 & & $\mathrm{C}$ & \\
\hline Manhattan Bch. F Sta 2 & MBF & -118.389 & 33.887 & Pleistocene medium alluvium & Qom & 4 & 414 & 85 & 308 & C & \\
\hline Lawndale LDS Church & LAW & -118.346 & 33.897 & Pleistocene fine alluvium & Qof & 4 & 364 & 46 & 265 & $\mathrm{C}$ & \\
\hline Arcadia & ARC & -118.059 & 34.127 & Holocene medium alluvium & Qym & 4 & 318 & 79 & 227 & $\mathrm{D}$ & \\
\hline Sandberg & SBM & -118.724 & 34.743 & Granitic rock & gr & 4 & 828 & 115 & & $\mathrm{~B}$ & \\
\hline Palm. Hwy 14 \& P Blvd & $\mathrm{P} 14$ & -118.135 & 34.581 & Holocene coarse alluvium & Qyc & 2 & 560 & & 485 & C & Palmdale $\mathrm{HI}$ site $67^{6}$ \\
\hline Arcadia Sch & ARS & -118.036 & 34.130 & Holocene medium alluvium & Qym & 4 & 318 & 79 & 362 & $\mathrm{D}$ & \\
\hline Inglewood Union Oll & IGU & -118.279 & 33.905 & Pleistocene medium alluvium & Qom & 4 & 414 & 85 & & $\mathrm{C}$ & \\
\hline Bell Gardens Church & $B G C$ & -118.158 & 33.965 & Holocene medium alluvium & Qym & 4 & 318 & 79 & 261 & $\mathrm{D}$ & \\
\hline Rolling Hills Estates & RHE & -118.356 & 33.787 & M-Late Miocene shale(Monterey) & $\mathrm{Tm}$ & 3 & 373 & & & $\mathrm{C}$ & avg sites $39^{4}, 40^{4}, 45^{4}, \operatorname{TAR}^{10}$ \\
\hline Littlerock B Canyon & LBC & -117.980 & 34.486 & Wthrd granodiorite & gr & 4 & 828 & 115 & & $\mathrm{~B}$ & \\
\hline Antelope Buttes & ANB & -118.361 & 34.758 & Wthrd granodiorite & gr & 4 & 828 & 115 & & $\mathrm{~B}$ & \\
\hline El Monte Ch 11338 & EMC & -118.019 & 34.093 & Holocene medium alluvium & Qym & 4 & 318 & 79 & 289 & $\mathrm{D}$ & \\
\hline Downey Cnty Maint & DOW & -118.167 & 33.924 & Holocene medium alluvium & Qym & 2 & 250 & & & $\mathrm{D}$ & \\
\hline Laguna Peak & LPK & -119.065 & 34.109 & Low-Miocene shale(Low-Topanga) & Ttlc & 3 & 556 & & & $\mathrm{C}$ & Morrison Cyn site $34^{1}$ \\
\hline
\end{tabular}




\begin{tabular}{|c|c|c|c|c|c|c|c|c|c|c|c|}
\hline Compton LDS Church & COM & -118.196 & 33.899 & Holocene medium alluvium & Qym & 4 & 318 & 79 & 185 & $\mathrm{D}$ & \\
\hline Baldwin Park Olive Sch & BPK & -117.974 & 34.100 & Holocene medium alluvium & Qym & 4 & 318 & 79 & 408 & $\mathrm{D}$ & \\
\hline Whittier & WHT & -118.029 & 34.015 & Up-Miocene sed rock(Puente) & $\mathrm{Tp}$ & 3 & 480 & & & C & avg Kagel ${ }^{10}$, Castaic ${ }^{6}$ \\
\hline Duarte Valley V Sch & DUA & -117.940 & 34.150 & Holocene coarse alluvium & Qyc & 4 & 448 & 47 & 485 & C & \\
\hline $\begin{array}{l}\text { Carson Del Amo Dolphin } \\
\text { Sch }\end{array}$ & CDA & -118.240 & 33.836 & Holocene medium alluvium & Qym & 1 & 190 & & 262 & $\mathrm{D}$ & \\
\hline Santa Fe Springs & SFS & -118.087 & 33.944 & Holocene medium alluvium & Qym & 4 & 318 & 79 & 350 & $\mathrm{D}$ & \\
\hline Carson C Ave Sch & CAS & -118.270 & 33.812 & Pleistocene fine alluvium & Qof & 4 & 364 & 46 & 198 & C & \\
\hline Neenach & NEE & -118.536 & 34.848 & Holocene medium alluvium & Qym & 4 & 318 & 79 & & $\mathrm{D}$ & \\
\hline West Covina & WCV & -117.952 & 34.064 & Holocene medium alluvium & Qym & 4 & 318 & 79 & 354 & $\mathrm{D}$ & \\
\hline Long Beach LC Libr & LBL & -118.194 & 33.840 & Pleistocene medium alluvium & Qom & 4 & 414 & 85 & & C & \\
\hline Port Hueneme N Lab & $\mathrm{PHN}$ & -119.206 & 34.145 & Holocene medium alluvium & Qyf & 2 & 326 & & & $\mathrm{D}$ & \\
\hline Rancho Palos Verdes & PVC & -118.396 & 33.746 & M-Late Miocene shale(Monterey) & $\mathrm{Tm}$ & 3 & 373 & & & C & avg sites $39^{4}, 40^{4}, 45^{4}, \operatorname{TAR}^{10}$ \\
\hline Ventura Holiday Inn & VHI & -119.293 & 34.276 & Holocene medium alluvium & Qym & 3 & 248 & & & $\mathrm{D}$ & Ventura Cty Gen site $26^{3}$ \\
\hline Baldwin Pk LDS Chu & СBP & -117.915 & 34.087 & Holocene medium alluvium & Qym & 4 & 318 & 79 & 347 & $\mathrm{D}$ & \\
\hline $\begin{array}{l}\text { Rancho P V Mira Catalina } \\
\text { Sch }\end{array}$ & RPV & -118.334 & 33.740 & M-Miocene sandst/siltst(Monterey) & $\mathrm{Tm}$ & 1 & 1038 & & 328 & $\mathrm{~B}$ & \\
\hline Huntington Beach & HBS & -118.044 & 33.727 & Holocene fine alluvium & Qyf & 4 & 240 & 56 & 203 & D & \\
\hline Long Beach City Hall & $\mathrm{LBCH}$ & -118.196 & 33.768 & Pleistocene medium alluvium & Qom & 1 & 372 & & & $\mathrm{C}$ & Magnolia site $1^{3}$ \\
\hline La Puente & LAP & -117.918 & 34.026 & Holocene medium alluvium & Qym & 4 & 318 & 79 & 285 & $\mathrm{D}$ & \\
\hline Hacienda Heights & HHT & -117.943 & 33.990 & Holocene medium alluvium & Qym & 4 & 318 & 79 & 278 & $\mathrm{D}$ & \\
\hline Lakewood & LWD & -118.099 & 33.846 & Holocene fine alluvium & Qyf & 4 & 240 & 56 & 230 & $\mathrm{D}$ & \\
\hline P. V. 1414 W 25th & SPP & -118.309 & 33.722 & M-Late Miocene shale(Monterey) & $\mathrm{Tm}$ & 3 & 373 & & & $\mathrm{C}$ & avg sites $39^{4}, 40^{4}, 45^{4}, \mathrm{TAR}^{10}$ \\
\hline Terminal I F Sta 112 & TMI & -118.269 & 33.736 & Holocene medium alluvium & Qym & 3 & 219 & & 260 & $\mathrm{D}$ & Terminal Is site $3^{3}$ \\
\hline Covina LDS Church & $\operatorname{ccs}$ & -117.871 & 34.078 & Holocene medium alluvium & Qom & 4 & 414 & 85 & 333 & C & \\
\hline La Habra & HBA & -117.973 & 33.921 & Pleistocene fine alluvium & Qof & 4 & 364 & 46 & 306 & C & \\
\hline Buena Park & BAP & -118.018 & 33.847 & Holocene medium alluvium & Qym & 4 & 318 & 79 & 220 & $\mathrm{D}$ & \\
\hline Seal Beach & SBO & -118.084 & 33.757 & Holocene fine alluvium & Qyf & 2 & 278 & & & $\mathrm{D}$ & \\
\hline Lancaster Airport & LNA & -118.214 & 34.739 & Pleistocene lacustrine & Qpl & 3 & 278 & & & $\mathrm{D}$ & Rosamond site $60^{6}$ \\
\hline Rosamond Airport & ROS & -118.206 & 34.870 & Pleistocene lacustrine & Qpl & 3 & 278 & & & $\mathrm{D}$ & Rosamond site $60^{6}$ \\
\hline Brea & BRA & -117.896 & 33.916 & Holocene medium alluvium & Qym & 4 & 318 & 79 & 289 & $\mathrm{D}$ & \\
\hline Wrightwood J Flat & WJF & -117.737 & 34.381 & Pelona Schist & ps & 4 & 828 & 115 & & $B$ & \\
\hline Garden Grove & GGS & -118.012 & 33.790 & Holocene fine alluvium & Qyf & 4 & 240 & 56 & 248 & $\mathrm{D}$ & \\
\hline Anaheim FSK Sch & $\mathrm{AHM}$ & -117.951 & 33.817 & Holocene fine alluvium & Qyf & 4 & 240 & 56 & 269 & $\mathrm{D}$ & \\
\hline Mt. Baldy Sch & MBS & -117.661 & 34.233 & Thin Hol alluvium/granite & Qyc-vc/gr & 3 & 800 & & & $\mathrm{~B}$ & Allen Ranch site $77^{7}$ \\
\hline Wrightwood S Valley & WSV & -117.658 & 34.369 & Holocene coarse alluvium & Qyc & 3 & 482 & & & $\mathrm{C}$ & Wrightwood site $76^{7}$ \\
\hline
\end{tabular}




\begin{tabular}{|c|c|c|c|c|c|c|c|c|c|c|c|}
\hline Villa Park CV Sch & VPS & -117.818 & 33.821 & Holocene medium alluvium & Qym & 4 & 318 & 79 & 614 & $\mathrm{D}$ & \\
\hline Huntington B LS F Sta & $\mathrm{HBF}$ & -117.997 & 33.662 & Pleistocene fine alluvium & Qof & 3 & 248 & & & $\mathrm{D}$ & avg 4 sites (LC) \\
\hline Rancho C Deer Cyn. & $\mathrm{RCM}$ & -117.579 & 34.169 & Granitic rock & gr & 4 & 828 & 115 & & $\mathrm{~B}$ & \\
\hline Rancho C Law Cntr & $\mathrm{RNC}$ & -117.574 & 34.104 & Holocene medium alluvium & Qym & 4 & 318 & 79 & & $\mathrm{D}$ & \\
\hline Featherly Park & FYP & -117.709 & 33.869 & Holocene medium alluvium & Qym & 4 & 318 & 79 & & $\mathrm{D}$ & \\
\hline Newport Beach C Hwy & $\mathrm{NBI}$ & -117.902 & 33.634 & Pleistocene medium alluvium & Qom & 4 & 414 & 79 & & $\mathrm{C}$ & \\
\hline Wrightwood Nielson R & WNR & -117.545 & 34.314 & Holocene coarse alluvium & Qyc & 3 & 482 & & & $\mathrm{C}$ & Wrightwood site $76^{7}$ \\
\hline Mojave Hwys 14 \& 58 & M58 & -118.175 & 35.070 & Holocene medium alluvium & Qym & 4 & 318 & 79 & & $\mathrm{D}$ & \\
\hline Tustin SC Sch & TUS & -117.824 & 33.728 & Holocene fine alluvium & Qyf & 4 & 240 & 56 & 242 & $\mathrm{D}$ & \\
\hline Phelan & PWR & -117.520 & 34.467 & Holocene medium alluvium & Qym & 4 & 318 & 79 & & $\mathrm{D}$ & \\
\hline Newport Beach & NNB & -117.931 & 33.623 & Pleistocene fine alluvium & Qof & 3 & 248 & & & $\mathrm{D}$ & avg 4 sites (LC) \\
\hline Receiving Sta & RST & -118.360 & 34.176 & Holocene coarse alluvium & Qyc & 1 & 332 & & & $\mathrm{D}$ & \\
\hline Brentwood VA Hosp. & BVA & -118.463 & 34.063 & Pleistocene medium alluvium & Qom & 1 & 421 & & & $\mathrm{C}$ & \\
\hline Wadsw. VA Hosp N & WVAN & -118.453 & 34.054 & Pleistocene medium alluvium & Qom & 1 & 389 & & & $\mathrm{C}$ & \\
\hline Wadsw. VA Hosp S & WVAS & -118.448 & 34.050 & Holocene medium alluvium & Qym & 1 & 410 & & & $\mathrm{C}$ & \\
\hline Monte Nido F Sta & MND & -118.693 & 34.078 & Miocene basaltic breccia & Tcvbp & 3 & 594 & & & $\mathrm{C}$ & avg sites $23^{1}, 28^{1}, 40^{2}, 57^{2}$ \\
\hline Malibu Epis Ch & MEC & -118.787 & 34.024 & Pleist terrace deps/Miocene shale & Qom/Tm & 3 & 373 & & 368 & $\mathrm{C}$ & avg sites $39^{4}, 40^{4}, 45^{4}, \mathrm{TAR}^{10}$ \\
\hline Hawthorne & HAW & -118.377 & 33.896 & Pleistocene medium alluvium & Qom & 2 & 326 & & & $\mathrm{D}$ & \\
\hline Pasadena Wilson Ave. & PSW & -118.127 & 34.136 & Pleistocene medium alluvium & Qom & 2 & 416 & & & C & CIT Athenaeum site $80^{7}$ \\
\hline Montebello SV Sch & MTL & -118.114 & 33.990 & Pleistocene medium alluvium & Qom & 4 & 414 & 85 & & $\mathrm{C}$ & \\
\hline Point Mugu NAS & PMG & -119.119 & 34.113 & Holocene fine alluvium & Qyf & 1 & 217 & & & $\mathrm{D}$ & \\
\hline Norwalk North 2 & NWN & -118.067 & 33.917 & Holocene medium alluvium & Qym & 4 & 318 & 79 & & $\mathrm{D}$ & \\
\hline Norwalk North 3 & NWN & -118.065 & 33.917 & Holocene medium alluvium & Qym & 4 & 318 & 79 & & $\mathrm{D}$ & \\
\hline Norwalk South & NWS & -118.067 & 33.915 & Holocene medium alluvium & Qym & 4 & 318 & 79 & & $\mathrm{D}$ & \\
\hline Littlerock Post Office & LRP & -117.991 & 34.522 & Holocene medium alluvium & Qym & 1 & 455 & & & $\mathrm{C}$ & \\
\hline Long Beach VA Hosp & VLB & -118.115 & 33.777 & Pleistocene medium alluvium & Qom & 1 & 364 & & & $\mathrm{C}$ & \\
\hline Brea Dam & BAD & -117.926 & 33.889 & Holocene medium alluvium & Qym & 2 & 350 & & & $\mathrm{D}$ & \\
\hline Diemer Filter Plant & DFL & -117.819 & 33.913 & Up-Miocene Puente fm & Tpsc & 3 & 480 & & & $\mathrm{C}$ & avg Kagel ${ }^{10}$, Castaic ${ }^{6}$ \\
\hline Huntington B Spd & HBS & -118.023 & 33.697 & Holocene fine alluvium & Qyf & 4 & 240 & 56 & 203 & $\mathrm{D}$ & \\
\hline Prado Dam & PRD & -117.640 & 33.888 & Holocene medium alluvium & Qym & 4 & 318 & 79 & & $\mathrm{D}$ & \\
\hline Santa Felicia Dam & SFD & -118.753 & 34.460 & M-Late Miocene shale(Monterey) & $\mathrm{Tm}$ & 3 & 397 & & & $\mathrm{C}$ & avg sites $39^{4}, 40^{4}, 45^{4}, \mathrm{TAR}^{10}$ \\
\hline Arcadia Forest Station & CF1 & -118.021 & 34.196 & Hol coarse alluv/granite & Qyc/gr & 4 & 828 & 115 & & $\mathrm{~B}$ & \\
\hline Whittier Narrows & WND & -118.054 & 34.031 & Holocene medium alluvium & Qym & 4 & 318 & 79 & & $\mathrm{D}$ & \\
\hline Leona Valley FS & LVF & -118.290 & 34.620 & Holocene medium alluvium & Qym & 1 & 323 & & & $\mathrm{D}$ & \\
\hline Long Beach Harbor & LBH & -118.200 & 33.754 & Holocene medium alluvium & Qym & 2 & 182 & & & $\mathrm{D}$ & \\
\hline
\end{tabular}




\begin{tabular}{|c|c|c|c|c|c|c|c|c|}
\hline Lancaster & LAN & -118.156 & 34.688 & Pleistocene lacustrine & Qpl & 3 & 278 & \\
\hline Valyermo Forest Sta & VYO & -117.851 & 34.444 & Holocene medium alluvium & Qym & 4 & 318 & 79 \\
\hline Paradise Springs & PSC & -117.805 & 34.397 & $<3 \mathrm{~m} \mathrm{Hol}$ coarse alluv/schist & sch & 4 & 828 & 115 \\
\hline Rosamond G Ranch & RGR & -118.265 & 34.827 & Pleistocene lacustrine & Qpl & 3 & 278 & \\
\hline Wheeler Gorge & $\begin{array}{l}W G \\
G\end{array}$ & -119.273 & 34.511 & $<5 \mathrm{~m} \mathrm{Hol} \mathrm{crs} \mathrm{al/} \mathrm{hard} \mathrm{Eocene} \mathrm{shale}$ & Tcwsh & 3 & 600 & \\
\hline Anacapa Island & ANI & -119.362 & 34.016 & Thin Hol alluv/granite & gr & 4 & 828 & 115 \\
\hline San Antonio Dam & SOD & -117.675 & 34.156 & Holocene coarse alluvium & Qyc & 4 & 448 & 47 \\
\hline Lockwood Valley & LWV & -119.131 & 34.749 & Holocene medium alluvium & Qym & 4 & 318 & 79 \\
\hline Costa Mesa FS & CM4 & -117.931 & 33.658 & Pleistocene fine alluvium & Qof & 3 & 248 & \\
\hline Cuddy Valley & CDY & -119.066 & 34.840 & Holocene medium alluvium & Qym & 4 & 318 & 79 \\
\hline Wrightwood PO & WTW & -117.629 & 34.360 & Holocene coarse alluvium & Qyc & 1 & 482 & \\
\hline Palmdale Black Butte & PBB & -117.728 & 34.586 & Quartz monzonite & qm & 4 & 828 & 115 \\
\hline Costa Mesa JW & JWA & -117.869 & 33.677 & Pleistocene fine alluvium & Qof & 2 & 248 & \\
\hline Newport Beach & NNB & -117.931 & 33.623 & Pleistocene medium alluvium & Qom & 4 & 414 & 85 \\
\hline Long Beach Recreation & LBR & -118.133 & 33.778 & Pleistocene medium alluvium & Qom & 2 & 364 & \\
\hline $\begin{array}{r}{ }^{*} \text { type } 1=\text { borehole meas } \\
\text { USGS and A } \\
\text { type } 1 \mathrm{~b}=\text { borehole mea } \\
\text { type } 2=\text { borehole meas } \\
\text { type } 3=\text { inferred from a } \\
\text { type } 4=\text { average velocit } \\
\text { type } 5=\text { surface-wave }\end{array}$ & $\begin{array}{l}\text { ment }( \\
\text { abian. } F \\
\text { ement } \\
\text { ment }(l \\
\text { age of b } \\
\text { or geolo } \\
\text { asureme }\end{array}$ & $\begin{array}{l}\text { SGS/Agbal } \\
\text { CDMG/NUF } \\
\text { SGS/Agbak } \\
\text { rehole mea } \\
\text { jic map unit } \\
\text { it }\end{array}$ & $\begin{array}{l}\text { ian) within } \\
\text { s the USG } \\
\text { EG/Crand } \\
\text { ian/CDMG } \\
\text { surements }\end{array}$ & $\begin{array}{l}300 \mathrm{~m} \text { of site (note: at some of these } \\
\text { value is shown in the first column, A } \\
\text { all) within } 300 \mathrm{~m} \text { of site } \\
\text { (NUREG/Crandall) within } 1500 \mathrm{~m} \text { of } \mathrm{s} \\
\text { in similar materials }\end{array}$ & abian in & eco & & \\
\hline \multicolumn{9}{|l|}{${ }^{1}$ USGS OFR 76-731 } \\
\hline \multicolumn{9}{|l|}{${ }^{2}$ USGS OFR 77-850 } \\
\hline \multicolumn{9}{|l|}{${ }^{3}$ USGS OFR 80-378 } \\
\hline \multicolumn{9}{|l|}{${ }^{4}$ USGS OFR 81-399 } \\
\hline \multicolumn{9}{|l|}{${ }^{5}$ USGS OFR 82-407 } \\
\hline \multicolumn{9}{|l|}{${ }^{6}$ USGS OFR 82-833 } \\
\hline \multicolumn{9}{|l|}{${ }^{7}$ USGS OFR 84-681 } \\
\hline \multicolumn{9}{|l|}{${ }^{8}$ USGS OFR 99-446 } \\
\hline \multicolumn{9}{|l|}{${ }^{9}$ USGS unpublished data } \\
\hline Agbabian & & & & & & & & \\
\hline
\end{tabular}




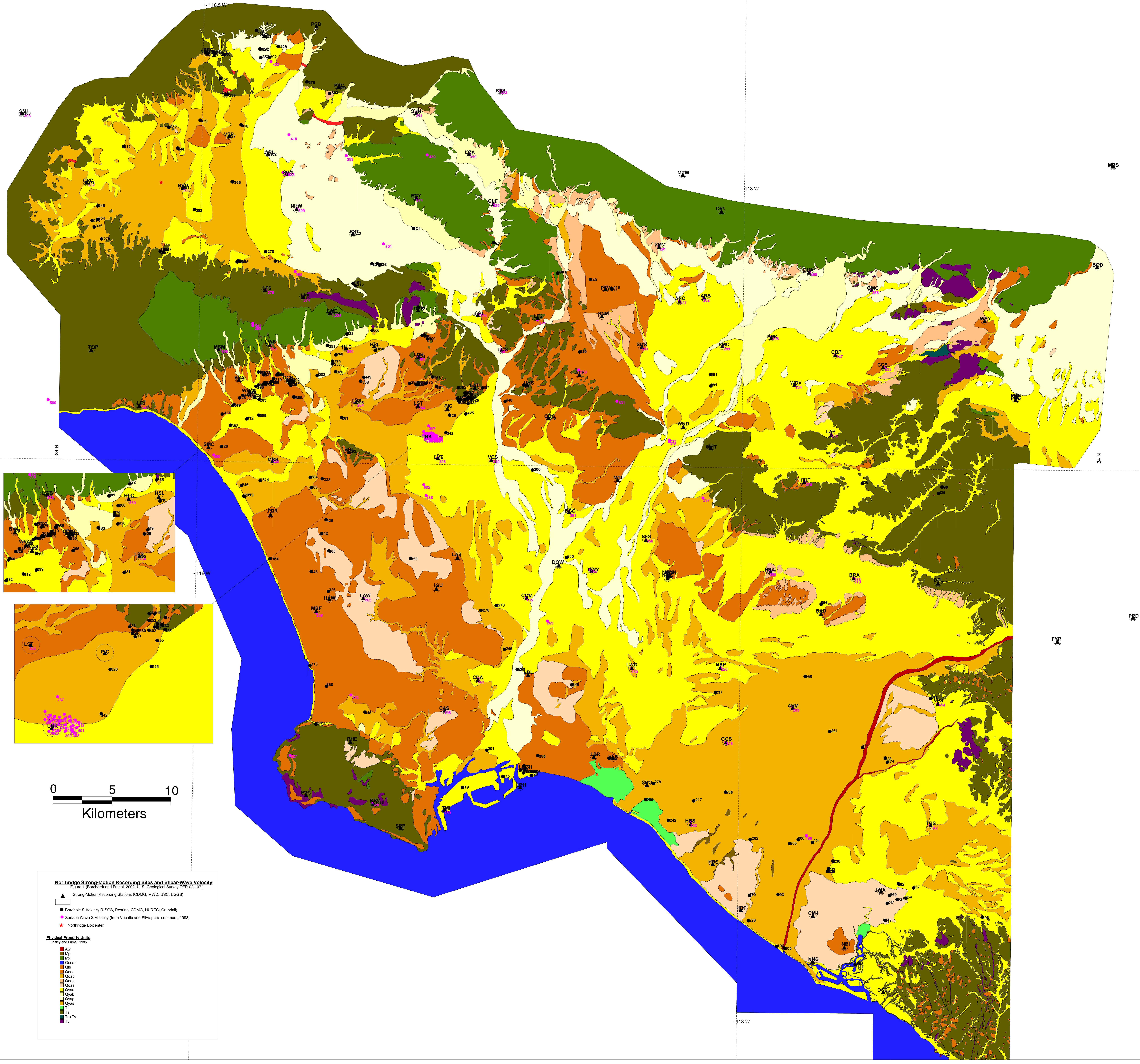




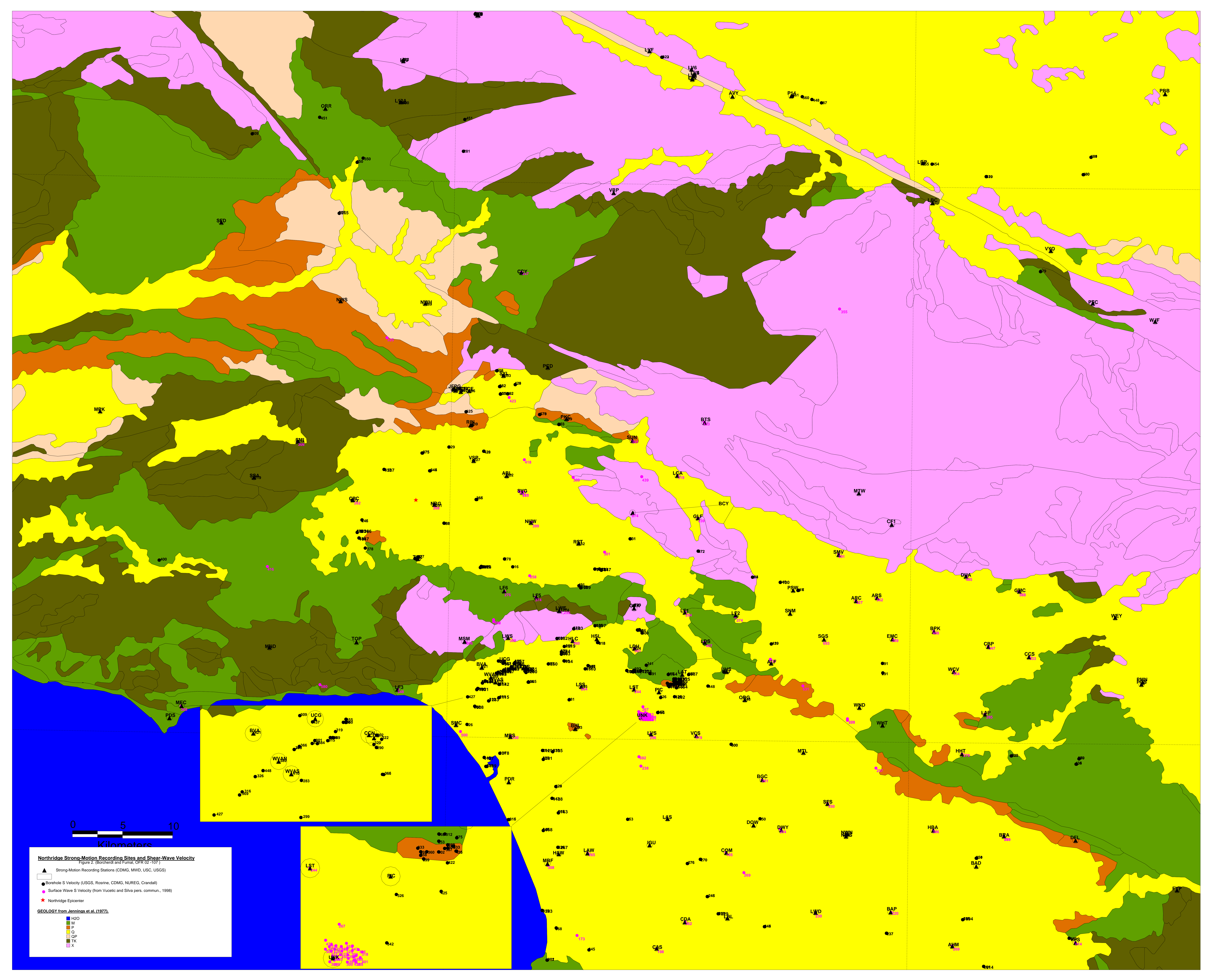



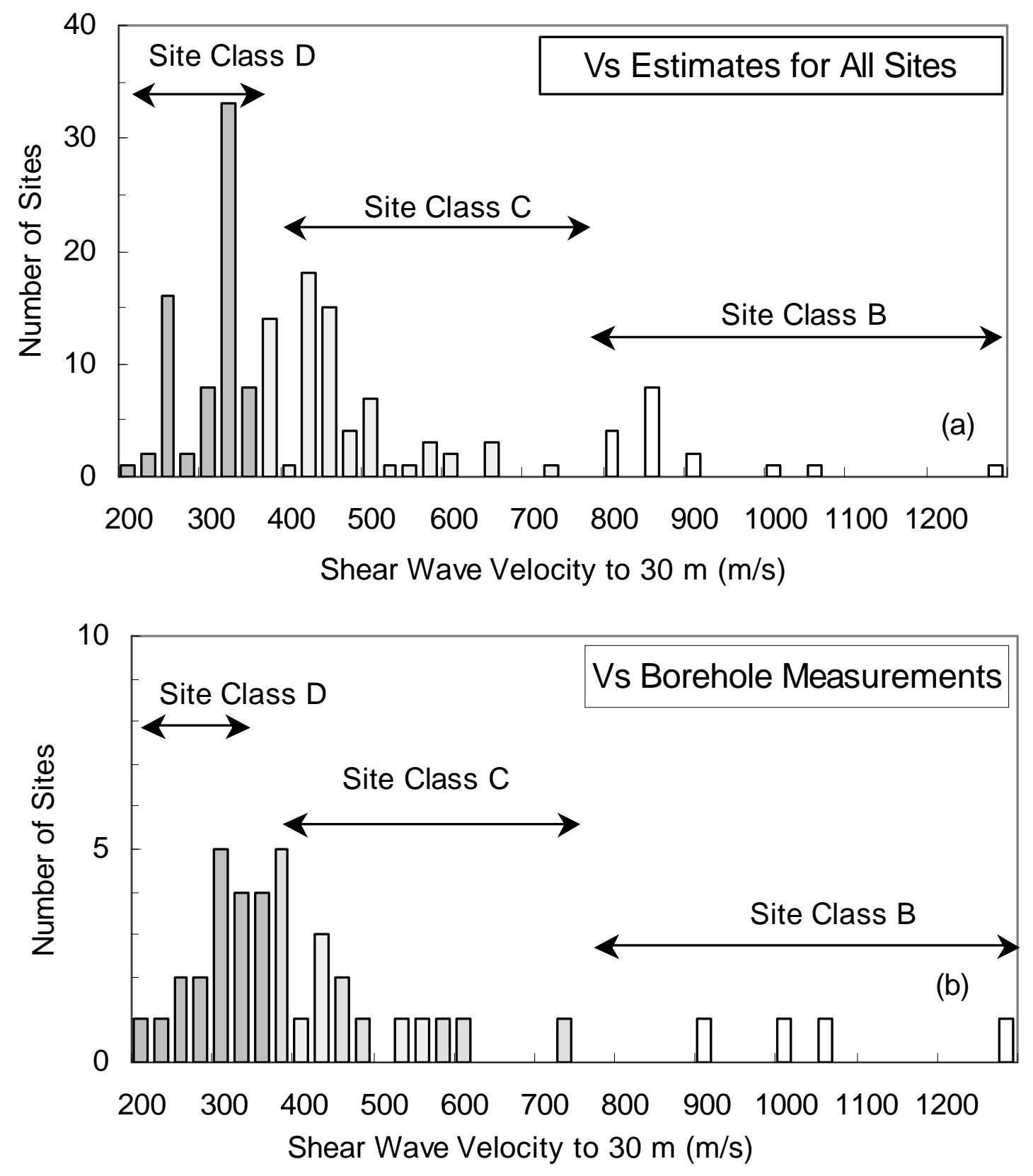

Figure 3. Histograms showing empirical distribution of shear-wave velocity estimates as inferred for each of the strong-motion recording sites (a) and for sites with borehole measurements (type 1 and 1b) as tabulated in Table 1. The distribution for all sites (a) suggests a tri-modal distribution centered at 337, 437, and $862 \mathrm{~m} / \mathrm{s}$. It shows that materials in site classes D, C, and B with shear velocities ranging between 200 and $1300 \mathrm{~m} / \mathrm{s}$ underlie the strong-motion recording sites in southern California. 


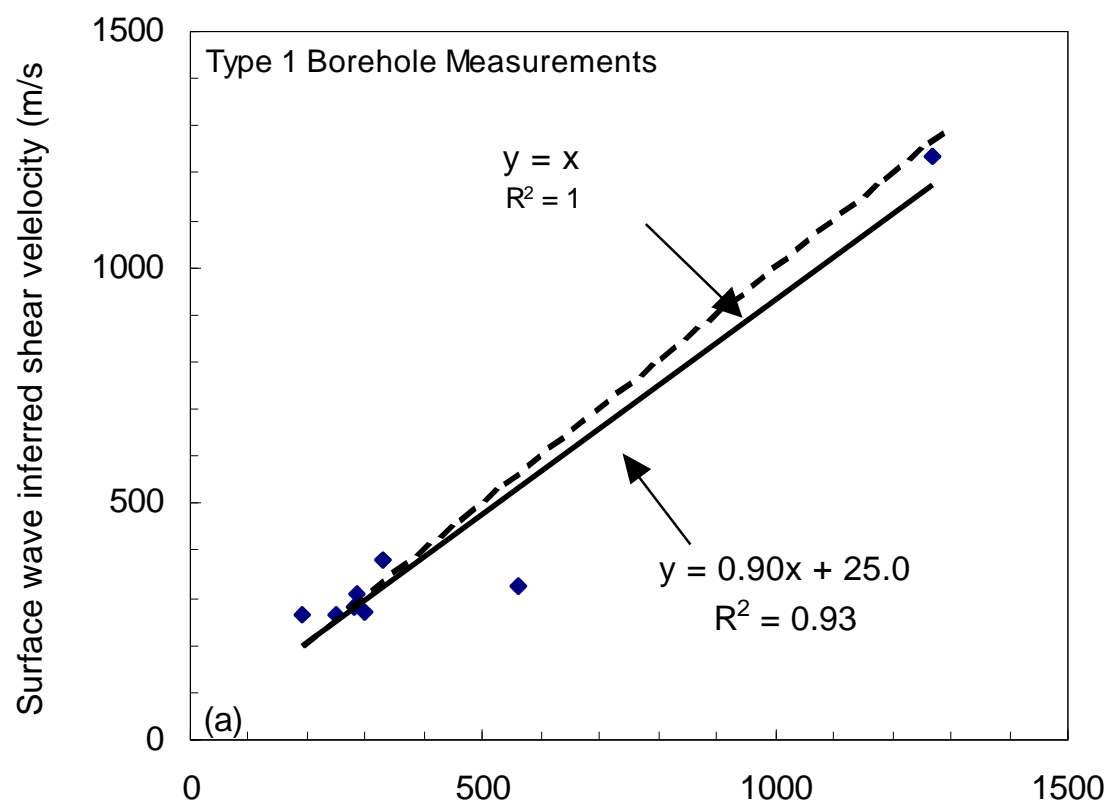

Borehole inferred shear velelocity $(\mathrm{m} / \mathrm{s})$

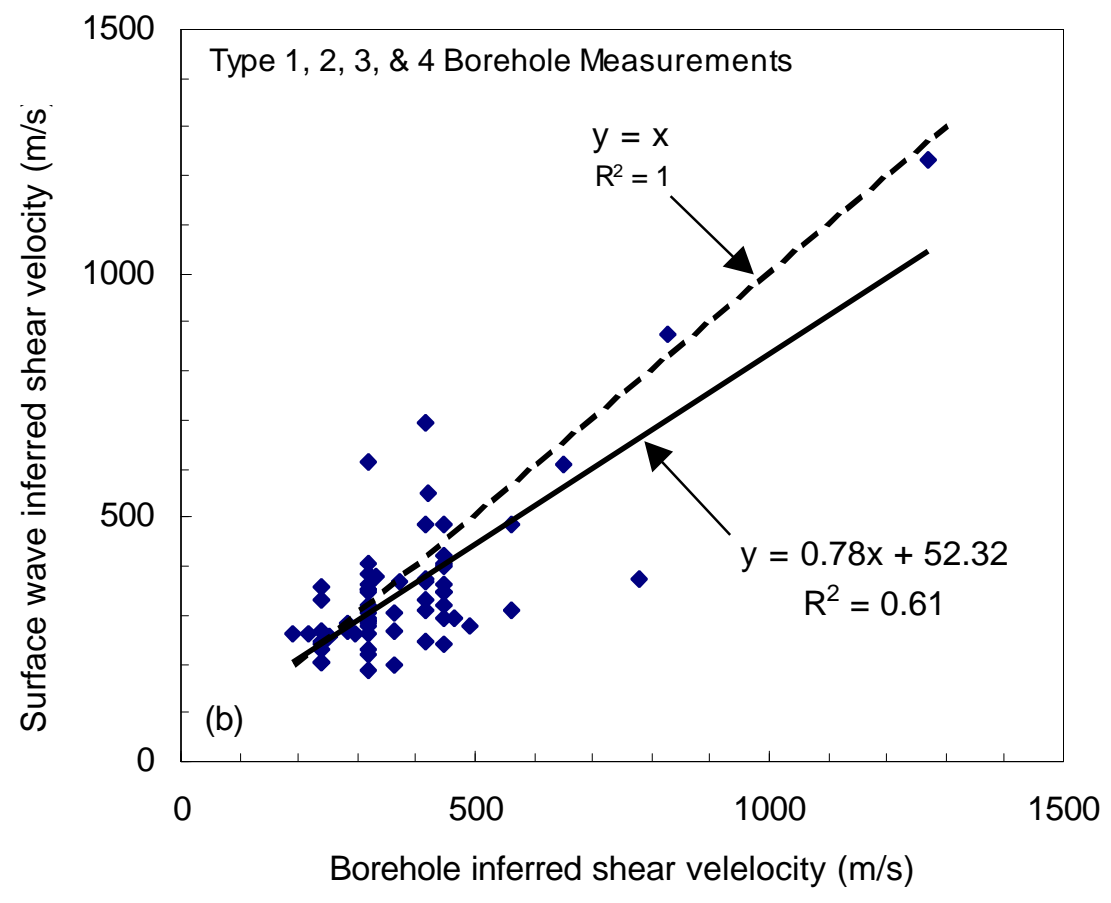

Figure 4. Estimates of shear-wave velocity inferred from surface-wave, shear-wave velocity measurements compared to those inferred from type-1 borehole measurements (a) and those inferred from type- 1, 2, 3, and 4 borehole information (b). 